\title{
Oleothorax: Review of Literature; Report of a Case Study and a Potential Treatment for Multi-Resistant Tuberculosis (MRTB)
}

\author{
Hassan Naji
}

\begin{abstract}
An 83-year-old female died of dementia with history of no previous disease. On dissection, paraffin was found in the right pleural cavity, indicated that the person received therapy for cavitary tuberculosis in the 1940s or1950s but no gross evidence of pulmonary or pleural tubercular disease was identified in the patient. Literature review on oleothorax indicated several reported complications of oleothorax. This case notes the lack of any apparent complications caused by oleothorax. In this paper, we present a case and outlines the importance of oleothorax in the medical history of tuberculosis, and as a potential treatment for multiresistant tuberculosis (MRTB).
\end{abstract}

Index Terms-Oleothorax, Pleuroliths, Tuberculosis, TB, Multi-Resistant Tuberculosis, MRTB, Paraffin Oil, Artificial Pneumothorax, Cavitary Tuberculosis, Purdue University.

\section{INTRODUCTION}

Oleothorax is a therapeutic method involving an intra- or extra-pleural injection of oil in pulmonary tuberculosis, mechanical expansion of lung, pleuropulmonary perforations, obliterative pneumothorax, and for artificial pneumothorax. Usually, the oil was removed after 18-24 months. However, many asymptomatic patients were lost to follow-up and their oil was never removed. Dr. Bernou was the first to establish the technique of oleothorax, by injecting a massive injection of intrapleural gomenol oil (Hochhegger, Zanetti, \& Marchiori, 2013). If it is used with judgment, it is a valuable complement of artificial pneumothorax. Later, for the prevention of recurrent tuberculous pneumothorax or persistent tuberculous empyema, oleothorax was used instead of thoracoplasty, because thoracoplasty is inadvisable in some patients due to debilities, poor prognosis, or advanced age (ANDREWS, MORTON, CURTIS, \& KLASSEN, 2012). While searching for a simple procedure that is minimally traumatic and is easily discontinued, Cournand and Berry (1942), used oleothorax in one case to prevent overdistension of the remaining lung and to maintain the mediastinum in midline. After one year, the maximum breathing capacity with one lung was almost the same to that obtained with two lungs (Cournand \& Berry, 1942).

From 1930's to 1950's, before the advent of antituberculosis drugs, oleothorax was widely accepted as the treatment for progressive tuberculosis. But due to several complications and after the advent of anti-tuberculosis drugs, the use of this therapy was abandoned. Long-term

Published on 29 August, 2019.

H. Naji is with Purdue University Northwest, USA. complications of oleothorax can occur if oil is not removed after instillation and include secondary infected pleuroliths, recurrent tuberculous empyema, pleural calcifications, oil embolism, fatty infiltrations of osseous structures, lipoid pneumonia, and chest wall abscess. Expansion of oleothorax may also occur many years after oil instillation due to irritating effect of instilled oil (Gillardin, Mortelé, Delens, \& Backer, 2013).

\section{CASE PRESENTATION}

An 83-year-old-female cadaver was inspected at Purdue University as an educational demonstration for students studying anatomy and physiology. The cadavers at Purdue University are acquired through the University of Cincinnati. University of Cincinnati, processes over 300 bodies per year and they distribute them to various educational institutions. University of Cincinnati acquire these cadavers form various nursing home facilities, coroner's office, and various healthcare settings. Purdue University staff transports the bodies into the University laboratories and use them for the fall, spring, and summer semesters. At the end of the year, the used cadavers are returned to University of Cincinnati so they can be returned back to families to carry their burial procedures. It is worth noting here that cadavers are treated with dignity and respect as every human cadaver enriches medical science and deserves to be treated with great respect. An oath is taken by students prior to dissecting cadavers to reflect on their conduct and habits in the dissection room. To bind science and humanity, it is our responsibility to respond to the anatomical gift of a human body with care, dignity, compassion, and respect (Gosh, S., 2017)

The cause of death of cadaver in this case was listed as dementia. No other medical history was provided. Only a copy of death certificate with dates of birth and death including apparent cause of death was received. On dissection, coagulated paraffin oil was found in the right pleural cavity (Figure 6). It was a unique finding in this case. Most people received this therapy for cavitary tuberculosis in the 1940s through 1950s but no gross evidence of pulmonary or pleural tubercular disease was identified in this patient. There was a marked regression in the right lung (Figure 3 ) where the paraffin oil was instilled and left for many years. The left lung (Figure 4) was well preserved with pink coloration as the oil was not instilled in this lung. The right lung was smaller than the left lung (Figure 2) in contrast to normal conditions in which right lung is larger than the left lung. The cadaver's heart (Figure 
5) showed normal size and texture indicating the lack of any symptoms associated with lung or cardiac disorders.

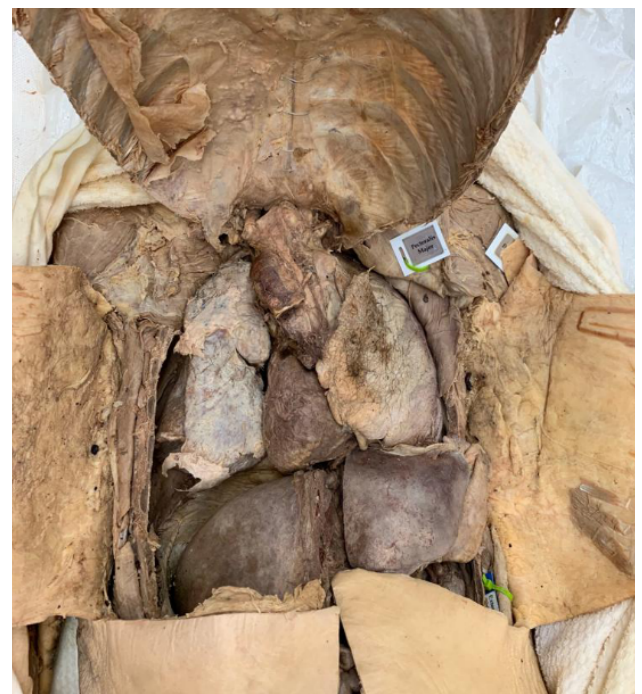

Figure 1.

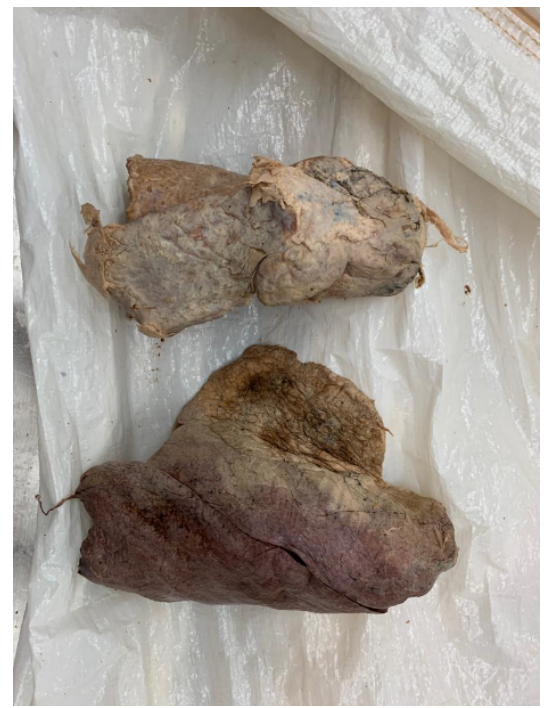

Figure 2.

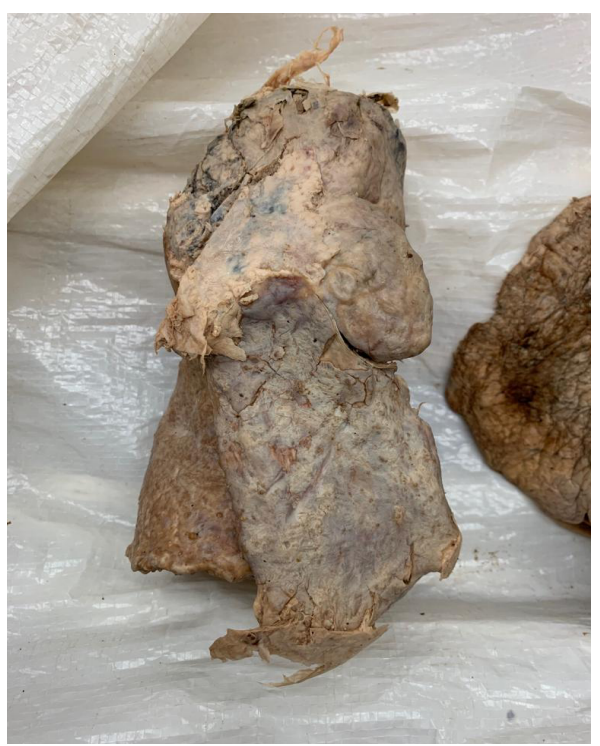

Figure 3

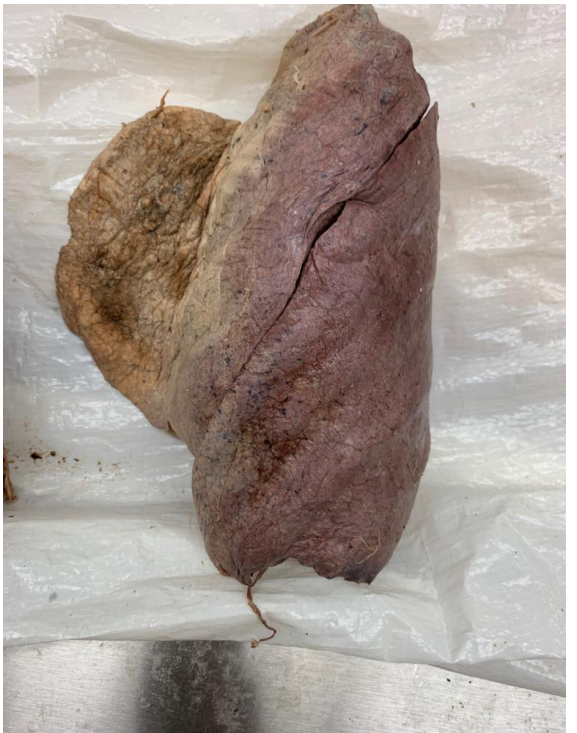

Figure 4.

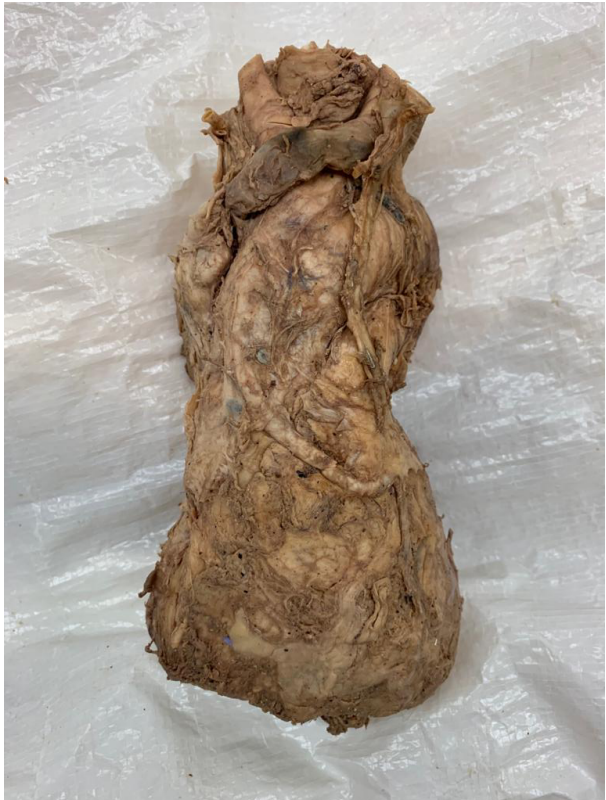

Figure 5.

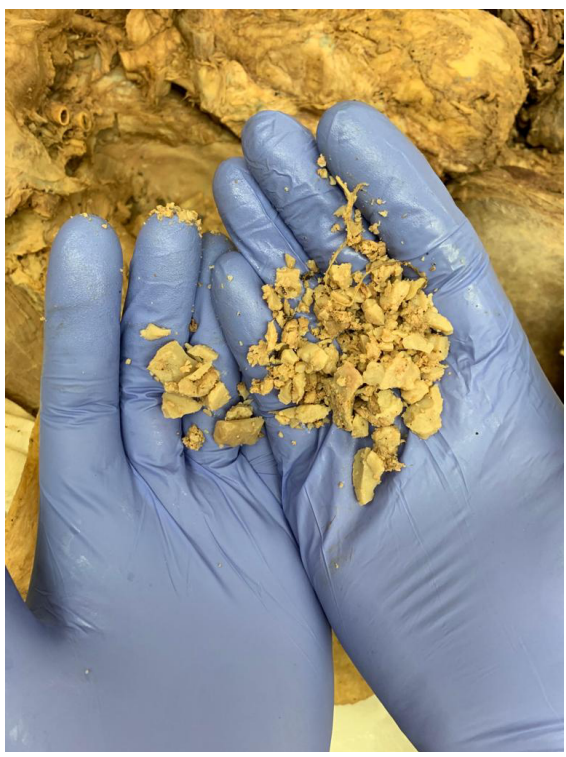

Figure 6. 


\section{DISCUSSION}

In several forms of tuberculosis, oleothorax has given satisfactory results than any other therapeutic method. These include:

- Pleuro-pulmonary perforations

- Mediastinal laxity combined with exaggerated pleural elasticity

- Obliterative pneumothorax

- Cases of ineffective collapse as a result of insufficient compression

- Pleurisies complicating pneumothorax

- Oleothoax as a substitute for artificial pneumothorax

Apart from the various advantages of this therapeutic process, certain defects and complications are also associated with it. We can avoid these problems by understanding the technique and the problems thoroughly. Oleothorax may cause febrile reactions of varying intensity between $99^{\circ}$ and $100^{\circ} \mathrm{F}$ to as high as $103^{\circ}$ and $104^{\circ} \mathrm{F}$ or more. These reactions vary among patients and depends mainly on the condition of the serous membrane at the time injection. The febrile reaction poses no danger if it is transitory, in fact, it gives useful information to the degree of pleural susceptibility. But, if the reaction is accompanied by effusion, it indicates that the pleura is irritated by the oil. The reaction may occur immediately or after several days (Hochhegger, Zanetti, \& Marchiori, 2013). There are several reported cases involving complications of oleothorax. And are reviewed as follows:

- An 84-year-old female presented with a persistent dry cough for 4 months. Her chest X-ray showed a mass in the right hemithorax and there was suspicion of lung cancer. So, the patient underwent CT, and the scans showed a posteriorly heterogenous mass in the upper right hemithorax. On aspiration, the viscous pleural oil was found to be mineral oil. While discussing medical history, the patient hardly remembered the oil injection for tuberculosis treatment about 60 years ago. The patient was diagnosed with unilateral oleothorax, that was initially considered as a lung mass, probably of neoplastic origin (Hochhegger, Zanetti, \& Marchiori, 2016). The complication aroused due to failure of removal of oil. The characteristic CT finding of oleothorax is an encapsulated pleural collection (Hochhegger, Zanetti, \& Marchiori, 2013).

- An 86-year-old woman presented with hypertension presented with burning chest and epigastric pain. She received therapy for pulmonary tuberculosis in the 1950s. She had no symptoms associated with acute coronary syndrome, and she was treated for gastroesophageal reflux, and her symptoms were relieved. A chest radiograph of the left lung showed a dense opacity in the upper area. The differential diagnosis for this abnormality includes an old hemothorax, calcified empyema and oleothorax. With the history of treatment with tuberculosis, the most probable diagnosis was oleothorax. But this patient had no complications or symptoms related to oleothorax (Koratala \& Bhatti, 2017).

- Three patients diagnosed with tuberculosis were treated with oleothorax 24, 34, and 36 years ago.
Two patients presented with atypical chest pain and one with chest wall and axillary pain on left side with a 'popping' sensation. Chest X-Rays films revealed bilateral and unilateral oleothorax with apparent fibrosisand scarring as a consequence of tuberculosis. On thoracotomy, focal herniation of paraffin was revealed in one patient. Upon culturing, the paraffin was removed from the thoracic cavity, grew Mycobacterium tuberculosis. In the other two patients, chest CT showed extrusion of oleothorax material but the presenting clinical symptoms were not felt to be associated with oleothorax (Kirshenbaum, Burke, Kirshenbaum, \& Cavallino, 1995).

The reviewed cases report several complications related to oleothorax. But in some cases, there were no complications associated with oleothorax. In our case there are also no complications linked to oleothorax. The patient lived well after being treated with oleothorax and ultimately died of dementia. Our case shows that if used properly, oleothorax can be used as an effective treatment for multiresistant tuberculosis (MRTB). Also, cases with oleothorax complications reported are limited indicating the lack of any unfavorable symptoms except for few cases. Many patients were successfully treated against tuberculosis using oleothorax without further complications. Such scanty reporting of complications associated with oleothorax, prompt us to think of using oleothorax as a potential treatment for MRTB.

In the early part of twentieth century, tuberculosis was the primary cause of death in age group of 15- to 40- years. The effective method of treatment was apical pulmonary collapse, initially performed by Hippocrates, on a pig's bladder. Recent practices of collapse therapy included oil and wax, air, silver mesh, muscle and breast flaps, Lucite spheres, rubber, gauze packs, and mammary prosthesis (Horowitz, Otero, Thurer, \& Bolooki 1992).

In 1882, Robert Koch identified the cause of tuberculosis, a bacterium named Mycobacterium tuberculosis. During sanatorium era, several therapeutic interventions were introduced. These included artificial pneumoperitoneum, plombage, phrenic nerve crush, artificial pneumothorax, and lung resection. Scientists started to find a cure of tuberculosis using chemotherapeutic agents and found streptomycin an effective anti-tuberculosis drug. But later on, it was found that streptomycin cause side effects of drug resistance. Scientists continued to search for more effective drugs against Mycobacterium tuberculosis and, then rifampin and isoniazid were developed. These drugs were effective and soon the cases of tuberculosis declined (Holm, 1959).

Poor public health infrastructure and decreased attention to tuberculosis led to recurrence of tuberculosis in 1980's and 1990's. Tuberculosis cases increased by about $20 \%$ in the United States between 1985 and 1992. A group of Mycobacterium tuberculosis develop resistance against isoniazid and rifampin and caused multiple drug-resistant tuberculosis. There are many problems associated with treatment of multiple drug-resistant tuberculosis and it is difficult to treat. Recently, new drugs named bedaquiline and delamanid are being used to treat multiple drug-resistant 
tuberculosis. (Murray, Schraufnagel, \& Hopewell, 2015).

The disease still remains a major global problem, with estimated 8.6 million new cases and 1.3 million deaths in 2012 alone. The major challenges associated with the treatment of tuberculosis include a significant rise of multiple drug-resistant tuberculosis, co-infection with human immunodeficiency virus (HIV), and vast reservoir of latently infected individuals. A major barrier to effective large-scale treatment of tuberculosis is the long duration use of chemotherapy (Ordonez et al., 2014).

We suggest the use of oleothorax as an adjunct therapy for treatment of multiple drug-resistant tuberculosis. As this treatment proved effective in the past without causing much complications, we can make interventions in the procedure and redevelop the process for control of MRTB.

\section{CONCLUSION}

Development of multiple drug-resistant tuberculosis is a major health problem in the globe nowadays. Scientists are remodeling drugs and searching for novel adjunctive therapies for the treatment of multiple drug-resistant tuberculosis. This case provides evidence for successful treatment of tuberculosis using oleothorax as a therapeutic method. It is important to find an effective cure for multiple drug-resistant tuberculosis as it causes many causalities. Further clinical studies and trials are advisable in order to effectively gauge the amount of oil needed.

\section{REFERENCES}

Andrews, N. C., Morton, D. R., Curtis, G. M., \& Klassen, K. P. (2012). Oleothorax Following Pulmonary Resection. Diseases of the Chest. https://doi.org/10.1378/chest.20.5.501

Cournand, A., \& Berry, F. B. (1942). The Effect of Pneumonectomy Upon Cardiopulmonary Function in Adult Patients. Annals of Surgery. https://doi.org/10.1097/00000658-194210000-00002

Gillardin, P., Mortelé, K. J., Delens, F., \& Backer, A. I. De. (2013). Images in clinical radiology: Pulmonary fat embolism. Jbr-Btr, 96(5), 319. Retrieved from http://www.embase.com/search/results?subaction=viewrecord\&from= export\&id=L372694002\%0Ahttp://sfx.library.uu.n1/utrecht?sid=EMB ASE\&issn $=17802393 \&$ id $=$ doi: $\&$ atitle $=$ Images + in + clinical + radiology $\% 3 \mathrm{~A}+$ Pulmonary+fat + embolism \&stitle $=$ JBR-BRT\&title $=$ JBRBTR\&volume $=96 \&$ is

Hochhegger, B., Zanetti, G., \& Marchiori, E. (2013). Oleothorax simulating pulmonary neoplasm. Annals of Thoracic Surgery. https://doi.org/10.1016/j.athoracsur.2012.09.076

Hochhegger, B., Zanetti, G., \& Marchiori, E. (2016). An uncommon chest mass: oleothorax. Jornal Brasileiro de Pneumologia. https://doi.org/10.1590/s1806-37562015000000217

Holm, J. (1959). How Can Elimination of Tuberculosis as a Public Health Problem be Achieved? American Review of Tuberculosis and Pulmonary Diseases. https://doi.org/10.1164/artpd.1959.79.5.690

Kirshenbaum, K. J., Burke, R. C., Kirshenbaum, M. D., \& Cavallino, R. P. (1995). Pleurocutaneous fistula as a complication of oleothorax CT findings in three patients. Clinical Imaging. https://doi.org/10.1016/0899-7071(94)00039-F

Koratala, A., \& Bhatti, V. (2017). Incidental Finding of Oleothorax. New England Journal of Medicine. https://doi.org/10.1056/NEJMicm1609176

Murray, J. F., Schraufnagel, D. E., \& Hopewell, P. C. (2015). Treatment of tuberculosis: A historical perspective. Annals of the American Thoracic Society. https://oi.org/10.1513/AnnalsATS.201509-632PS

Ordonez, A. A., Maiga, M., Gupta, S., Weinstein, E. A., Bishai, W. R., \& Jain, S. K. (2014). Novel Adjunctive Therapies for the Treatment of Tuberculosis. Current Molecular Medicine. https://doi.org/10.2174/1566524013666131118112431 